\title{
Well-differentiated papillary mesothelioma of the peritoneum is genetically defined by mutually exclusive mutations in TRAF7 and CDC42
}

\author{
Meredith Stevers ${ }^{1} \cdot$ Joseph T. Rabban $^{1} \cdot$ Karuna Garg $^{1} \cdot$ Jessica Van Ziffle ${ }^{1,2} \cdot$ Courtney Onodera $^{2} \cdot$ \\ James P. Grenert ${ }^{1,2} \cdot$ Iwei Yeh $^{1,2} \cdot$ Boris C. Bastian ${ }^{1,2} \cdot$ Charles Zaloudek $^{1} \cdot$ David A. Solomon $\mathbb{D}^{1,2}$
}

Received: 15 June 2018 / Revised: 28 July 2018 / Accepted: 28 July 2018 / Published online: 31 August 2018

(c) United States \& Canadian Academy of Pathology 2018

\begin{abstract}
Well-differentiated papillary mesothelioma is an uncommon mesothelial neoplasm that most frequently arises in the peritoneal cavity of women of reproductive age. Whereas malignant mesothelioma is an aggressive tumor associated with poor outcome, well-differentiated papillary mesothelioma typically exhibits indolent behavior. However, histologically differentiating between these two entities can be challenging, necessitating the development of distinguishing biomarkers. While the genetic alterations that drive malignant mesothelioma have recently been determined, the molecular pathogenesis of well-differentiated papillary mesothelioma is unknown. Here we performed genomic profiling on a cohort of ten welldifferentiated papillary mesothelioma of the peritoneum. We identified that all tumors harbored somatic missense mutations in either the TRAF7 or CDC42 genes, and lacked alterations involving BAP1, NF2, CDKN2A, DDX3X, SETD2, and ALK that are frequent in malignant mesothelioma. We recently identified that another mesothelial neoplasm, adenomatoid tumor of the genital tract, is genetically defined by somatic missense mutations in the TRAF7 gene, indicating a shared molecular pathogenesis between well-differentiated papillary mesothelioma and adenomatoid tumors. To the best of our knowledge, well-differentiated papillary mesothelioma is the first human tumor type found to harbor recurrent mutations in the CDC42 gene, which encodes a Rho family GTPase. Immunohistochemistry demonstrated intact BAP1 expression in all cases of well-differentiated papillary mesothelioma, indicating that this is a reliable marker for distinguishing well-differentiated papillary mesothelioma from malignant mesotheliomas that frequently display loss of expression. Additionally, all welldifferentiated papillary mesothelioma demonstrated robust expression of L1 cell adhesion molecule (L1CAM), a marker of NF-kB pathway activation, similar to that observed in adenomatoid tumors. In contrast, we have previously shown that L1CAM staining is not observed in normal mesothelial cells and malignant mesotheliomas of the peritoneum. Together, these studies demonstrate that well-differentiated papillary mesothelioma is genetically defined by mutually exclusive mutations in TRAF7 and CDC42 that molecularly distinguish this entity from malignant mesothelioma.
\end{abstract}

Electronic supplementary material The online version of this article (https://doi.org/10.1038/s41379-018-0127-2) contains supplementary material, which is available to authorized users.

David A. Solomon

david.solomon@ucsf.edu

1 Department of Pathology, University of California, San Francisco, CA, USA

2 Clinical Cancer Genomics Laboratory, University of California, San Francisco, CA, USA

\section{Introduction}

Well-differentiated papillary mesothelioma (commonly abbreviated WDPM) is a relatively uncommon subtype of mesothelial neoplasm that is most frequently found in women of reproductive age, but can also be seen in men and postmenopausal women [1-7]. Unlike malignant mesothelioma, well-differentiated papillary mesothelioma is not associated with prior asbestos exposure. Well-differentiated papillary mesothelioma most commonly arises in the peritoneal cavity, but can also be found in the pleural cavity, pericardium, and tunica vaginalis $[8,9]$. These tumors are usually discovered incidentally during surgical procedures for other clinical indications, often in close proximity to the associated 
principal pathology. Histologically, well-differentiated papillary mesothelioma are an exophytic papillary neoplasm lined by a single layer of cuboidal mesothelial cells with bland nuclear cytology and very low to absent mitotic activity. Typically, they are solitary lesions that lack architectural complexity and stromal invasion. Well-differentiated papillary mesothelioma express markers typical of mesothelial cells including calretinin, WT1, D2-40, and cytokeratin 5/6. Welldifferentiated papillary mesothelioma typically follow an indolent or benign clinical course, in contrast to malignant mesothelioma that is a highly aggressive tumor associated with poor outcome. However, there are several reported cases of well-differentiated papillary mesothelioma behaving in a malignant fashion, with dissemination leading to patient mortality $[4,7,10]$. Whether such cases represent malignant transformation of well-differentiated papillary mesothelioma or instead represent misclassified malignant mesotheliomas is unknown. These tumor entities can often be reliably differentiated in resection specimens based on the presence of nuclear atypia, mitotic activity, and complex architecture in malignant mesotheliomas that are not found in welldifferentiated papillary mesothelioma [11-13]. However, differentiating between these two entities in small biopsy or cytology specimens can be challenging given their shared mesothelial cell of origin and lack of distinguishing biomarkers. Therefore, it is critical to identify such biomarkers that help to differentiate well-differentiated papillary mesothelioma from malignant mesothelioma and better predict outcome for patients with mesothelial neoplasms.

There is currently controversy in the literature regarding the true biologic nature of well-differentiated papillary mesothelioma, and no defining molecular alterations have been identified to date to prove that these are indeed clonal neoplasms. Some authors have hypothesized that welldifferentiated papillary mesothelioma represents reactive mesothelial hyperplasia, given that these lesions are often found adjacent to another principal pathology or in patients with a prior history of surgery [14]. Other authors consider well-differentiated papillary mesothelioma to be a neoplasm of uncertain malignant potential and have treated patients with adjuvant chemotherapy upon diagnosis $[2,7]$. Here we performed genomic analysis on a cohort of ten welldifferentiated papillary mesothelioma of the peritoneum in order to assess the molecular pathogenesis of this poorly understood mesothelial neoplasm.

\section{Materials and methods}

\section{Study population and tumor specimens}

This study was approved by the Institutional Review Board of the University of California, San Francisco. Ten cases of well-differentiated papillary mesothelioma of the peritoneum were retrieved from the pathology archives of our institution, spanning years 1993 to 2014. All tumor specimens had been fixed in $10 \%$ neutral-buffered formalin and embedded in paraffin. Paraffin-embedded blocks of uninvolved normal tissue (e.g., lymph node or gastrointestinal biopsy) were also retrieved for nine of the ten patients. Pathologic review of all tumor samples was performed to confirm the diagnosis by a group of expert gynecologic pathologists (J.T.R., K.G., C.Z., and D.A.S.).

\section{Targeted next-generation DNA sequencing and mutational analysis}

Tumor tissue and uninvolved normal tissue was macrodissected from formalin-fixed, paraffin-embedded blocks for the 10 patients. Genomic DNA was extracted from the macrodissected tissue using the QIAamp DNA FFPE Tissue Kit (Qiagen) according to the manufacturer's protocol. Capture-based next-generation DNA sequencing was performed at the UCSF Clinical Cancer Genomics Laboratory as previously described using an assay that targets all coding exons of 479 cancer-related genes, TERT promoter, select introns and upstream regulatory regions of 47 genes to enable detection of structural variants including gene fusions, and DNA segments at regular intervals along each chromosome to enable genome-wide copy number and zygosity analysis, with a total sequencing footprint of $2.8 \mathrm{Mb}$ (UCSF500 Cancer Panel; Supplementary Table 1) $[15,16]$. Sequencing libraries were prepared from genomic DNA, and target enrichment was performed by hybrid capture using a custom oligonucleotide library (Roche NimbleGen). Sequencing was performed on an Illumina HiSeq 2500. Duplicate sequencing reads were removed computationally to allow for accurate allele frequency determination and copy number calling. The analysis was based on the human reference sequence (NCBI build 37) using the following software packages: BWA, Samtools, Picard tools, GATK, CNVkit, Pindel, SATK, Annovar, Freebayes, and Delly. Single nucleotide variants, insertions/deletions, and structural variants were visualized and verified using the Integrated Genome Viewer. Genomewide copy number analysis based on on-target and off-target reads was performed by CNVkit and Nexus Copy Number (Biodiscovery).

\section{Immunohistochemistry for BAP1 and L1CAM}

Immunohistochemistry was performed on whole formalinfixed, paraffin-embedded tissue sections of nine of the ten cases of well-differentiated papillary mesothelioma. Immunohistochemistry for BAP1 was performed using a mouse monoclonal anti-BAP1 antibody (clone C-4, Santa Cruz Biotechnology, sc-28383) at 1:100 dilution following 


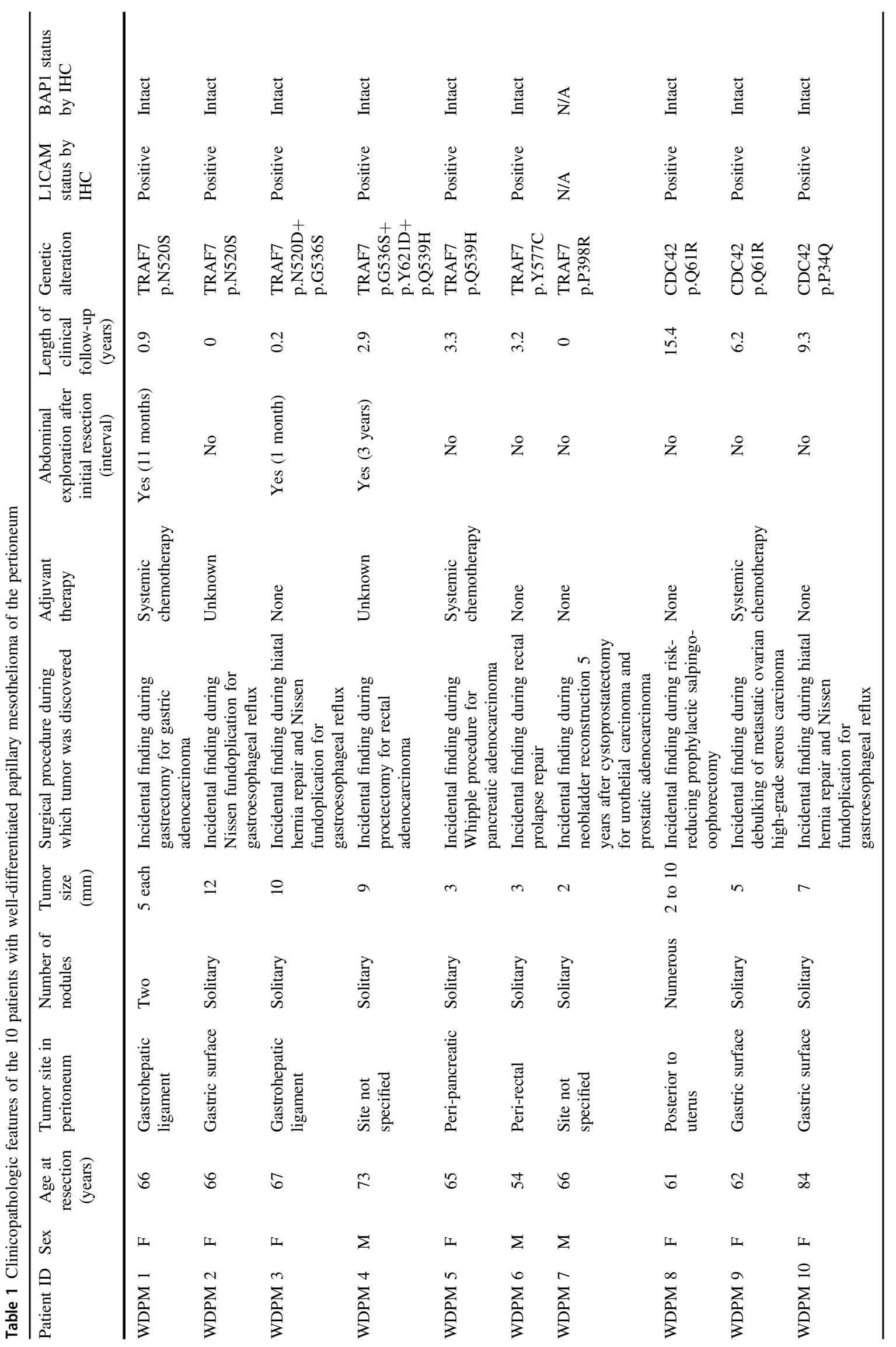


Fig. 1 Well-differentiated papillary mesothelioma of the peritoneum harbors frequent somatic mutations of the TRAF7 gene. a, b Hematoxylin and eosin stained sections of WDPM \#2 excised from the gastric surface of a 66-year-old woman after incidental discovery during Nissen fundoplication for gastroesophageal reflux. c Nextgeneration sequencing reads from the tumor and uninvolved normal gastric tissue demonstrating a somatic TRAF7 p.N520S missense mutation (c. 1559A >G)
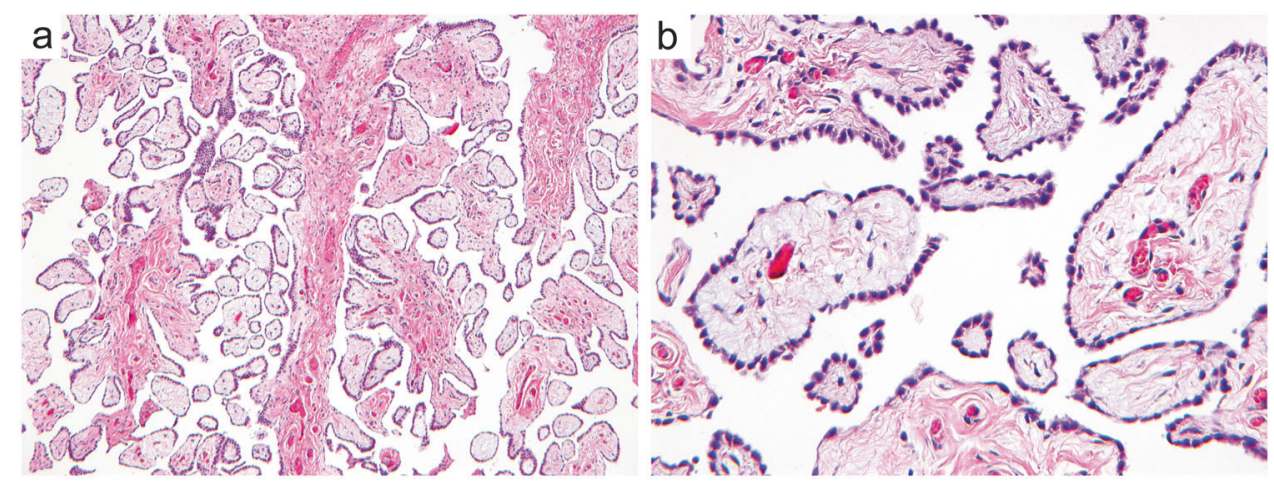

C $2,225,550 \mathrm{bp}$ $2,225,560 \mathrm{bp}$

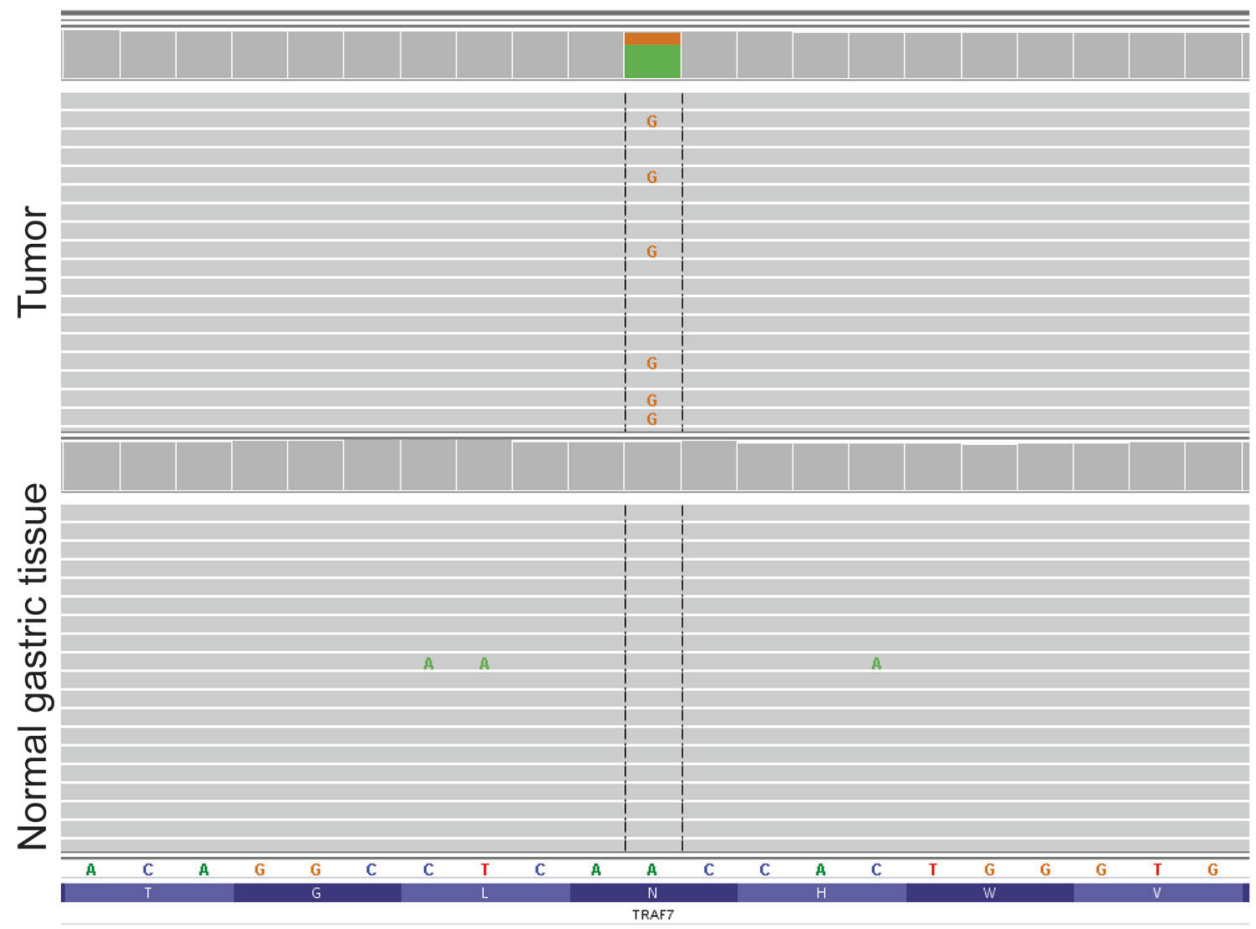

ER2 antigen retrieval in a Leica Bond automated stainer. Immunohistochemistry for L1CAM was performed using a mouse monoclonal anti-L1CAM antibody (clone UJ127.11, Sigma, L4543) at 1:1800 dilution following antigen retrieval in a Ventana Benchmark automated stainer. Diaminobenzidine was used as the chromogen, followed by hematoxylin counterstain. BAP1 staining was scored as either intact (strong homogeneous nuclear staining of $>90 \%$ of tumor cells) or lost (no nuclear staining in tumor cells with intact expression in non-neoplastic stromal and endothelial cells). No cases showed a mosaic staining pattern for BAP1. L1CAM staining was scored as either positive (membranous staining on a majority of tumor cells) or negative (absence of staining). No cases showed L1CAM staining in only a minority of the tumor cells. The BAP1 immunohistochemical staining results shown in Fig. 6 for normal mesothelial cells, adenomatoid tumor of the genital tract, and malignant peritoneal mesothelioma were previously reported by Joseph et al. [15]. The L1CAM immunohistochemical staining results shown in Fig. 6 for normal mesothelial cells, adenomatoid tumor of the genital tract, and malignant peritoneal mesothelioma were previously reported by Goode et al. [16].

\section{Results}

\section{Clinical features of the well-differentiated papillary mesothelioma patient cohort}

In order to study the molecular pathogenesis of well-differentiated papillary mesothelioma, we assembled a 
Fig. 2 Well-differentiated papillary mesothelioma of the peritoneum harbors somatic $C D C 42$ mutations in those cases lacking TRAF7 alterations. a, b Hematoxylin and eosin stained sections of WDPM \#10 excised from the gastric surface of an 84-year-old woman after incidental discovery during Nissen fundoplication for gastroesophageal reflux. c Nextgeneration sequencing reads from the tumor and uninvolved normal gastric tissue demonstrating a somatic $C D C 42$ p.P34Q missense mutation (c.101C $>$ A)
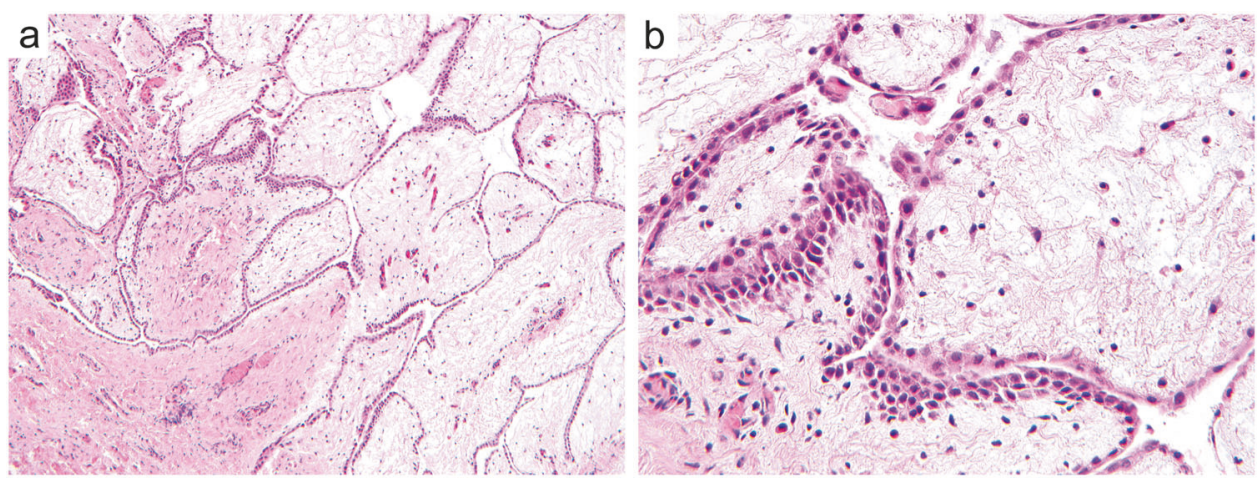

C

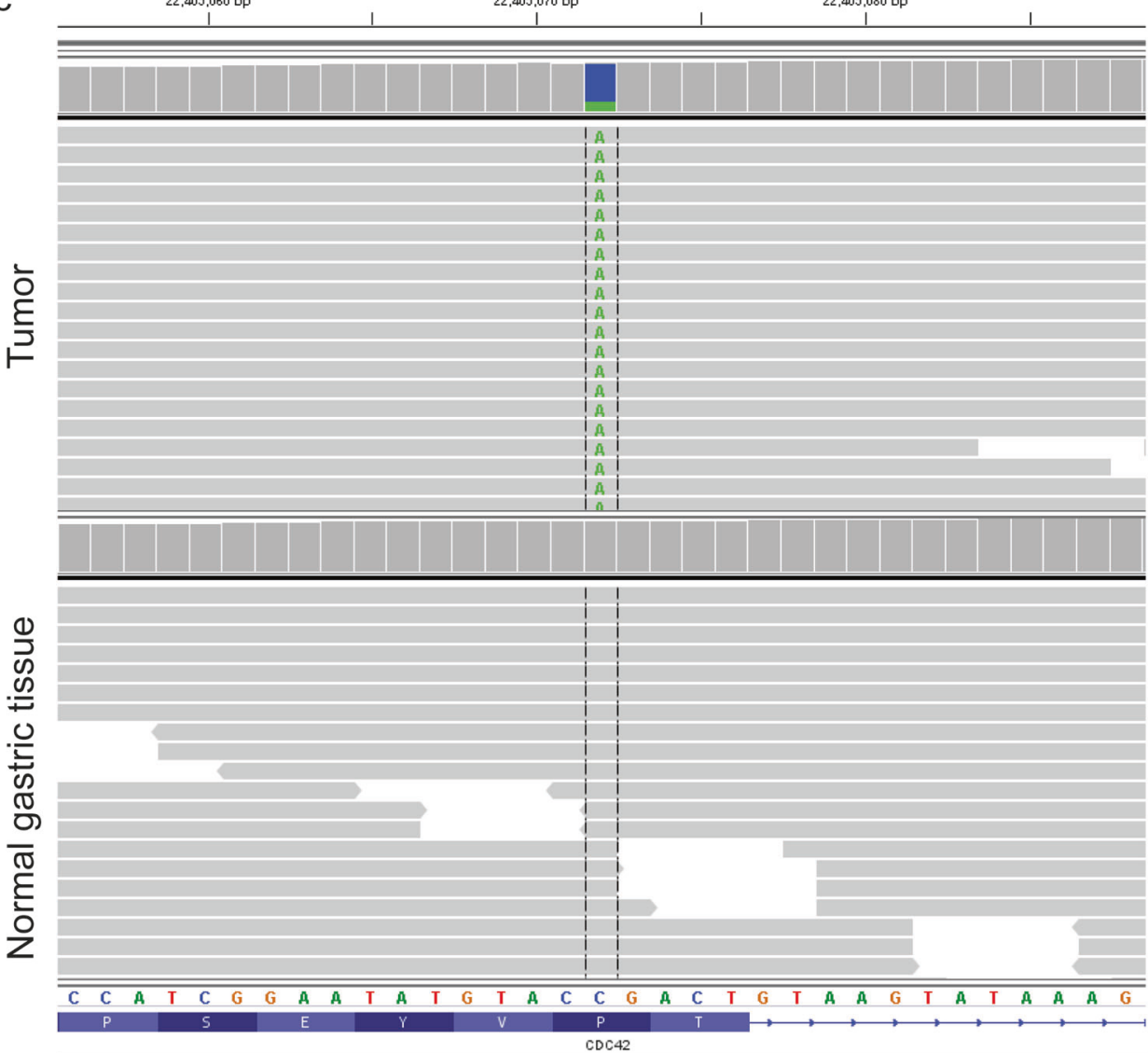

cohort of tumor and matched normal tissue from ten patients for genomic analysis. The clinical features of this patient cohort are listed in Table 1. The three male and seven female patients ranged in age from 54 to 84 years (median 66 years). All of the tumors were located in the peritoneum and were incidentally found during surgical procedures for other clinical indications including Nissen fundoplication for gastroesophageal reflux disease, rectal prolapse repair, prophylactic salpingo-oophorectomy, and gastrectomy for adenocarcinoma of the stomach. In all cases, the tumors were identified in the immediate vicinity of the principal pathology being surgically repaired or excised. For example, tumors were located on the gastric surface or gastrohepatic ligament of patients undergoing Nissen fundoplication or were on the serosal surface of the neobladder in a patient with a history of cystectomy for invasive urothelial carcinoma. Eight of the patients harbored solitary tumors ranging in size from 2 to $12 \mathrm{~mm}$. One of the patients (WDPM \#1) harbored two distinct tumor nodules both $\sim 5 \mathrm{~mm}$ that were found in close proximity on the gastrohepatic ligament during gastrectomy for signet ring cell adenocarcinoma. Another patient (WDPM \#8) harbored several tumor nodules ranging from 2 to $10 \mathrm{~mm}$ that were found in close proximity along the posterior pelvic 
Fig. 3 Oncoprint summary table of the 10 patients with well-differentiated papillary mesothelioma of the peritoneum. Patient age, sex, number of tumor nodules, TRAF7 and CDC42 mutation status, number of additional somatic mutations, quantity of chromosomal copy number alterations, and immunohistochemical staining results for L1CAM and BAP1 are shown

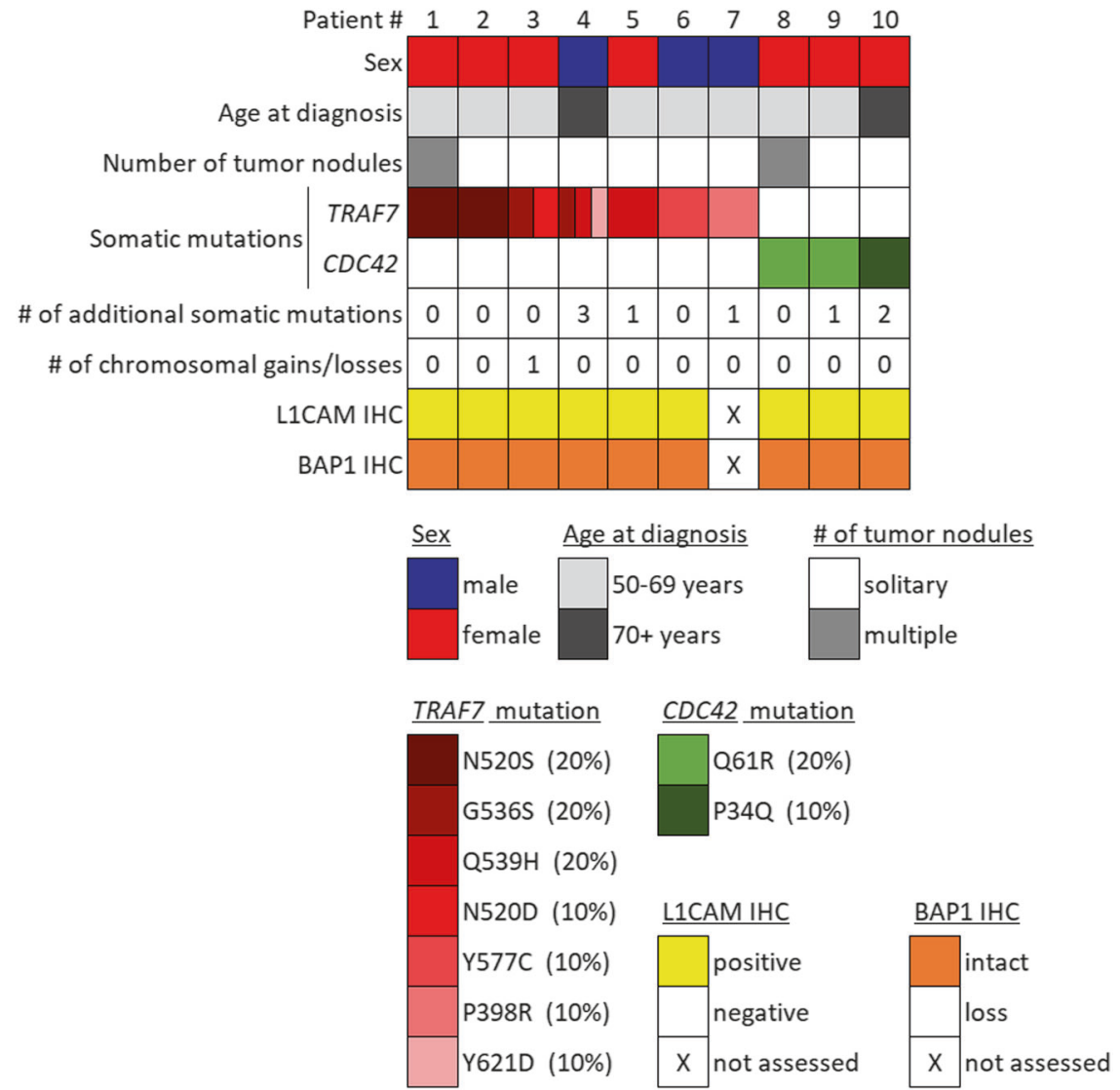

wall during risk-reducing salpingo-oophorectomy. None of patients received any adjuvant therapy directed at the well-differentiated papillary mesothelioma beyond surgical excision, but three of the patients did receive systemic chemotherapy for other indications. None of the patients in this cohort experienced disease recurrence after a median clinical follow-up of 3.1 years, which included three patients who underwent subsequent abdominal exploration after tumor excision.

\section{Histologic features of the well-differentiated papillary mesothelioma cohort}

Histologic features of the ten tumors are shown in Supplementary Figure 1. All cases consisted of an exophytic papillary proliferation of mesothelial cells with central fibrovascular cores containing a variable amount of collagenous and edematous stroma. The papillary cores were lined by a single layer of cuboidal mesothelial cells and uniformly lacked nuclear atypia and any appreciable mitotic activity. In three cases, immunohistochemical stains for calretinin, WT1, and cytokeratin $5 / 6$ had been performed as part of the diagnostic workup and were all positive in the tumor cells, supporting the diagnosis of a mesothelial neoplasm (data not shown).

\section{Genomic analysis of well-differentiated papillary mesothelioma}

Targeted next-generation sequencing was performed on genomic DNA isolated from the ten tumors and matched normal tissue as described in the Methods. This identified somatic missense mutations in the TRAF7 gene or CDC42 gene in all ten cases (Figs. 1 and 2, Supplementary Table 2). Mutations in TRAF7 and CDC42 were mutually exclusive, with seven cases that harbored TRAF7 mutations and were $C D C 42$ wild type, while the remaining three cases harbored CDC42 mutations and were TRAF7 wild type (Fig. 3). These TRAF7 and CDC42 missense mutations were verified to be somatic (i.e., tumor-specific) in all cases with available matched normal tissue $(n=9)$. Snapshots of the TRAF7 and CDC42 mutations are shown in Supplementary Figure 2.

TRAF7 encodes an E3 ubiquitin ligase and is a member of the family of tumor necrosis factor alpha receptorassociated factors (TRAFs). Somatic mutations in the TRAF7 gene have been recently identified as a defining genetic feature of adenomatoid tumors of the genital tract [16], and are also frequent in perineuriomas and meningiomas [17-19]. Similar to the TRAF7 mutations observed in these tumor types, the TRAF7 mutations in welldifferentiated papillary mesothelioma were all missense 
Fig. 4 Diagram of the human TRAF7 protein with locations of the somatic missense mutations identified in the 10 cases of welldifferentiated papillary mesothelioma. Also shown are locations of the missense mutations in the 31 adenomatoid tumors of the genital tract recently reported by Goode et al. [16]. The mutations all cluster within the WD40 repeats at the $\mathrm{C}$ terminus of the protein

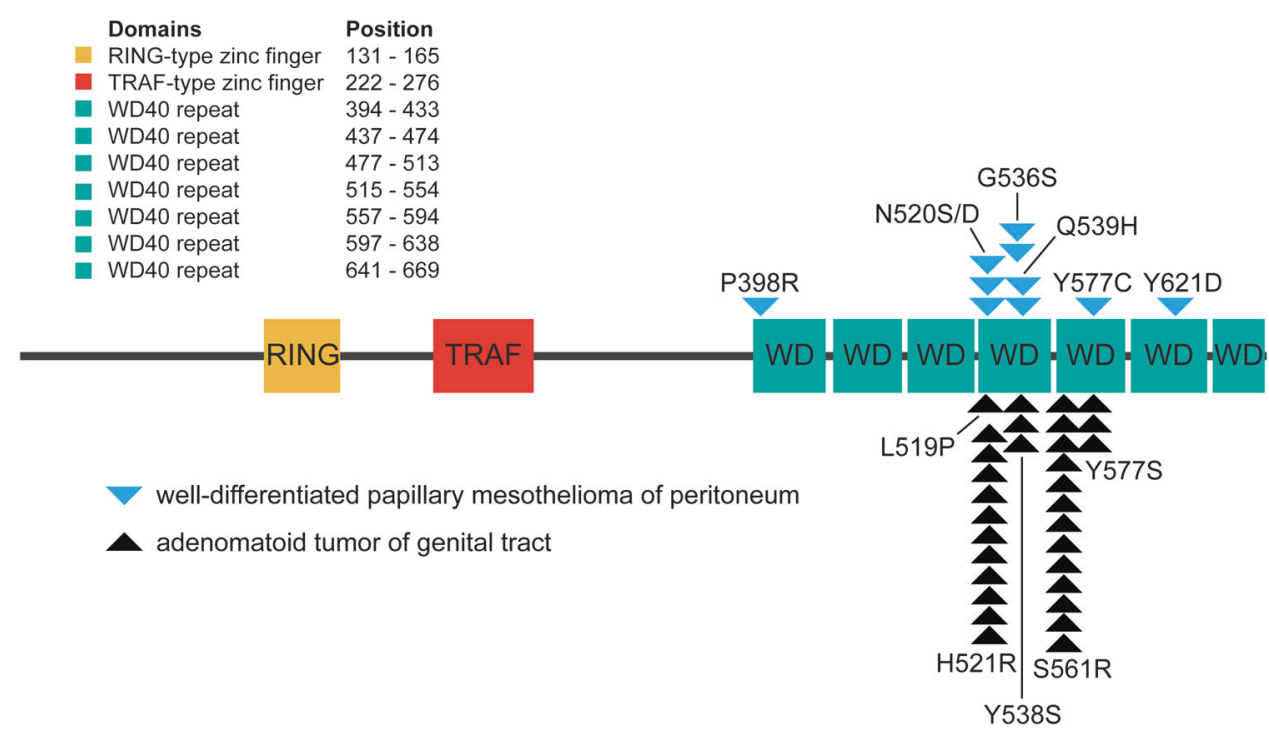

mutations that clustered in the WD40 repeats at the $\mathrm{C}$ terminus of the protein (Fig. 4). In five of the seven cases harboring TRAF7 mutations, a single missense mutation was present. WDPM \#3 harbored a focal amplification of the TRAF7 gene on chromosome 16p13 and two TRAF7 missense mutations (p.N520D and p.G536S) that were either present in trans (on opposite alleles) or within separate tumor subclones. WDPM \#4 harbored three TRAF7 missense mutations (p.G536S, p.Q539H, and p.Y621D; Supplementary Figure 2). The p.G536S and p.Q539H mutations were present either in trans or within separate subclones, while the p.Y621D mutation was too distant to phase. In addition to the recurrent TRAF7 mutations found in well-differentiated papillary mesothelioma, the presence of a focal amplification in one case and multiple mutations in two cases indicates a strong selection pressure for the acquisition of TRAF7 alterations during the development of well-differentiated papillary mesothelioma. The variant allele frequencies for the TRAF7 mutations ranged from 4-45\%. Cases with the highest TRAF7 variant allele frequencies had genomic DNA isolated from areas histologically visualized to contain a high tumor cell content, whereas cases with lower allele frequencies had genomic DNA that was isolated from tissue visualized to contain a lesser tumor to normal cell content. These data suggest that TRAF7 mutations were likely heterozygous and clonal (i.e., present in all tumor cells), indicating that they are probably an early or initiating event in well-differentiated papillary mesothelioma tumorigenesis.

CDC42 encodes a Rho family GTPase belonging to the Ras superfamily of GTPases. Two well-differentiated papillary mesothelioma cases harbored the identical p.Q61R mutation in $C D C 42$, while a third case harbored p.P34Q mutation. Similar to the TRAF7 mutations, the variant allele frequencies for the $C D C 42$ mutations (ranging from 20-38\%) indicated that these are also likely to be clonal heterozygous alterations in well-differentiated papillary mesothelioma and are probably early or initiating genetic events in those well-differentiated papillary mesothelioma lacking TRAF7 alterations. The p.Q61R mutation is located within a GTP binding domain, whereas the p.P34Q mutation is located within the effector domain (Fig. 5a). Amino acid sequence of CDC42 was aligned with the related Rho family GTPases RAC1 and RHOA, as well as the homologous Ras family GTPases (NRAS, HRAS, and KRAS), which are all known to be recurrently mutated in human cancers (Fig. 5b). Mutational hotspots in each of these proteins was annotated using data from the Catalogue of Somatic Mutations in Cancer (COSMIC) database [version 84 release]. Recurrent mutations in the CDC42 gene have not been previously reported in any human tumor type to the best of our knowledge, and the p.Q61R and p.P34Q variants in CDC42 are not present in the COSMIC database. However, the p.Q61 residue of CDC42 located in the highly conserved GTP binding domain is the equivalent residue to p.Q61 in the NRAS, HRAS, and KRAS proteins, which is a mutational hotspot with thousands of confirmed somatic mutations in human cancers present in the COSMIC database. The p.P34 residue in the effector domain of CDC42 where a mutation was identified in WDPM \#10 does not correspond to a mutational hotspot in any of the other Rho or Ras family GTPases.

In the two patients with multiple distinct tumor nodules (WDPM \#1 and 8), genomic DNA was pooled from the multiple nodules to ensure adequate sequencing depth. In both of these tumors, only a single TRAF7 mutation or $C D C 42$ mutation was identified, respectively. This indicates that the multiple tumor nodules that were found in close proximity were likely to share a common ancestral clone, rather than representing multiple distinct tumors. 
a

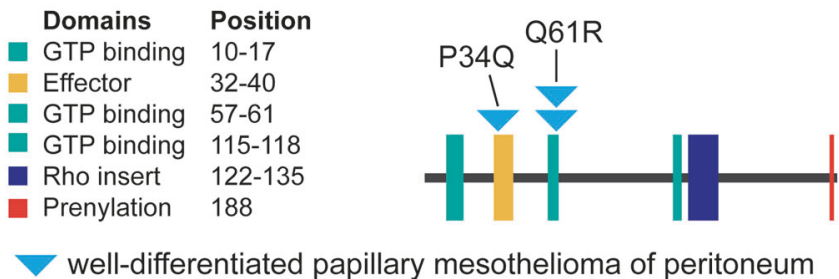

b

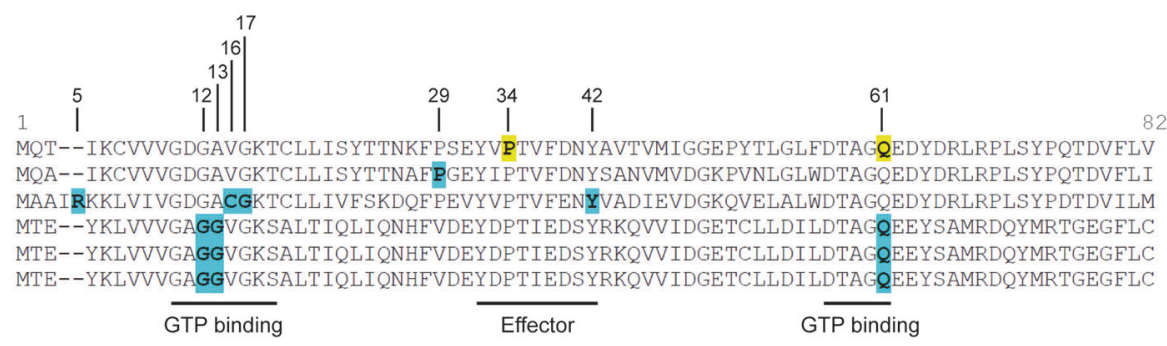

Fig. 5 a Diagram of the human CDC42 protein with locations of the somatic missense mutations identified in the 10 cases of welldifferentiated papillary mesothelioma. The mutations are present within GTP binding and effector domains of the protein. b Amino acid sequence from the $\mathrm{N}$-terminus of $\mathrm{CDC} 42$, the related Rho family GTPases RAC1 and RHOA, and the homologous Ras family GTPases. Residues that are recurrently mutated in human cancers are highlighted in blue, including the p.Q61 mutational hotspot in NRAS, HRAS, and

Additionally, the TRAF7 and CDC42 mutations were the solitary somatic alterations identified in these two cases, indicating that these well-differentiated papillary mesothelioma did not acquire accompanying genetic alterations among the assayed genes to explain the multifocal growth.

Beyond TRAF7 and CDC42, none of the other 477 sequenced genes contained recurrent somatic mutations in the ten well-differentiated papillary mesothelioma. While five cases contained additional somatic nonsynonymous mutations in addition to TRAF7 or $C D C 42$, all of these additional mutations are considered variants of unknown significance, as they do not affect genes known to be important in mesothelioma tumorigenesis and are not mutations that have been previously seen before in human cancers (COSMIC database, version 84 release). The complete list of somatic single nucleotide variants and insertions/deletions identified in the well-differentiated papillary mesothelioma cohort is shown in Supplementary Table 2. The total somatic mutation burden was low in all tumors (uniformly less than 2 somatic mutations per megabase). Chromosomal copy number analysis revealed trisomy 7 and focal amplification of chromosome 16p13.3 containing the TRAF7 gene in WDPM \#3 (Supplementary Table 3). None of the other nine cases harbored chromosomal gains, losses, or focal amplifications or deletions. All tumors lacked identifiable mutations, deletions, or rearrangements involving the $B A P 1, C D K N 2 A, N F 2, D D X 3 X$, SETD2, TP53, and $A L K$ genes known to be recurrently
KRAS. The residues in CDC42 found to harbor somatic mutations in well-differentiated papillary mesothelioma are highlighted in yellow. These include the p.Q61 residue (mutated in two cases) located in the highly conserved GTP binding domain that is the equivalent residue to the p.Q61 mutational hotspot in the Ras family proteins. The p.P34 residue (mutated in one case) located in the effector domain does not correspond to a mutational hotspot in any of other Rho or Ras family GTPases

altered in pleural or peritoneal malignant mesotheliomas $[15,20-23]$.

\section{BAP1 and L1CAM immunohistochemical assessment of well-differentiated papillary mesothelioma}

The BAPl tumor suppressor gene has emerged as a frequent target of inactivating mutations, deletions, or rearrangements in malignant mesothelioma, particularly in peritoneal cases where genetic alterations and associated loss of nuclear BAP1 immunostaining are seen in $>80 \%$ of cases according to multiple case series [15, 24-29]. In contrast, we have previously documented intact BAP1 expression by immunohistochemistry in normal mesothelial cells of the peritoneum, adenomatoid tumors of the genital tract $(n=16)$, and well-differentiated papillary mesothelioma of the peritoneum $(n=6)$ [15]. Here we find intact BAP1 expression in all nine of the well-differentiated papillary mesothelioma cases in this cohort with tissue available for immunohistochemical evaluation (Fig. 6).

TRAF7 belongs to the family of TNFo receptorassociated factors that are known to regulate the nuclear factor-kappa B (NF-kB) signaling pathway [30]. We previously demonstrated that tumor-derived TRAF7 mutations induce activation of NF-kB signaling and cause increased expression of L1 cell adhesion molecule (L1CAM) [16], a known transcriptional target of NF-kB that has emerged as a reliable surrogate marker for tumors with activated NF-kB 


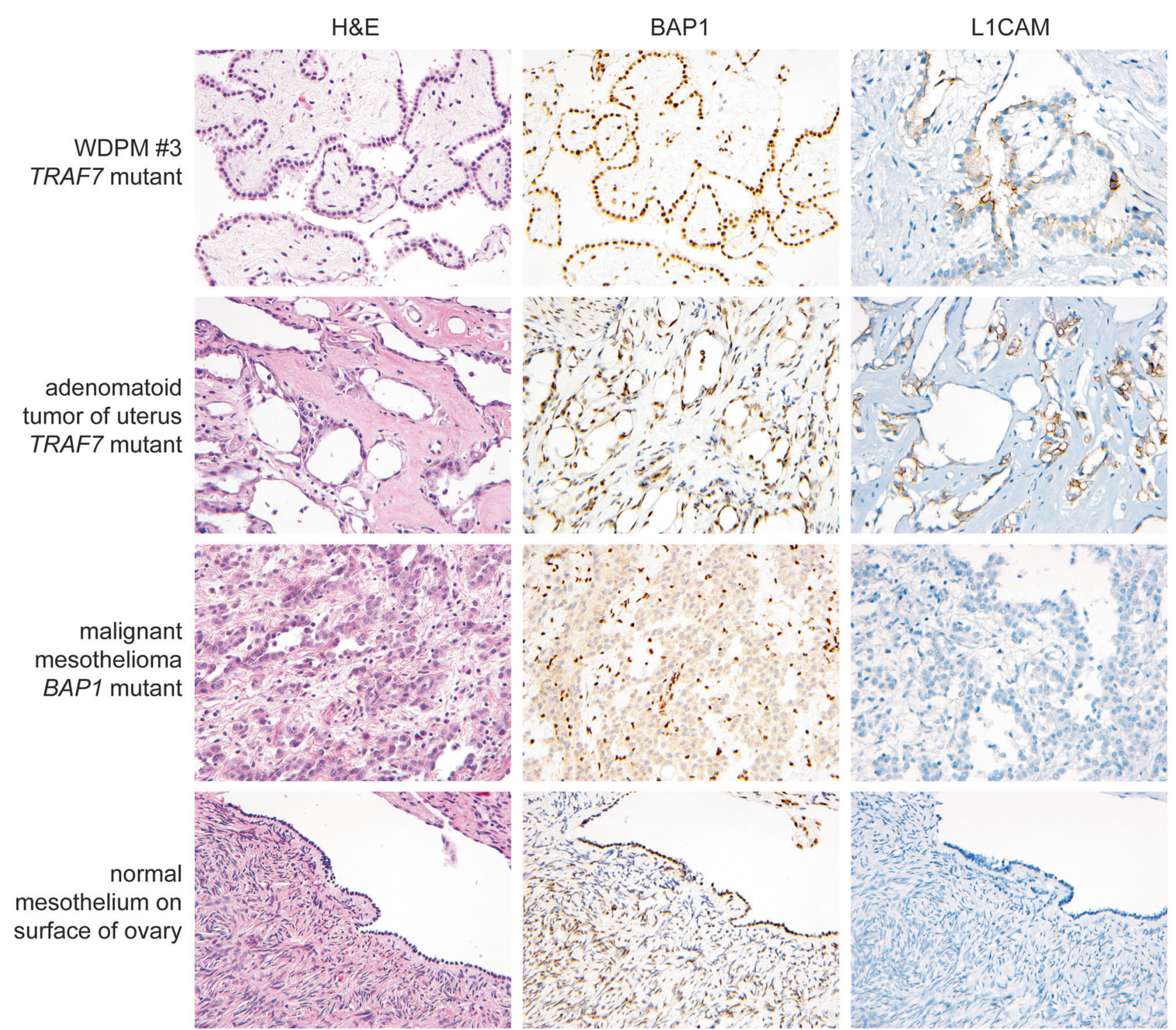

Fig. 6 Immunohistochemistry for BAP1 and L1 cell adhesion molecule (L1CAM) on mesothelial neoplasms of the peritoneum. BAP1 staining shows intact/retained expression in all cases of well-differentiated papillary mesothelioma of the peritoneum and adenomatoid tumors of the genital tract, with frequent somatic loss of expression in malignant

signaling $[31,32]$. Here we find strong membranous staining for L1CAM protein in all nine of the welldifferentiated papillary mesothelioma cases in this cohort with tissue available for immunohistochemical evaluation (Fig. 6), which include both TRAF7 and CDC42 mutant cases. This was comparable to the L1CAM expression we previously documented in adenomatoid tumors of the genital tract harboring TRAF7 mutations $(n=8)$, whereas no appreciable L1CAM staining was seen in normal mesothelial cells lining organs of the peritoneal cavity $(n=7)$, nor was staining seen in malignant peritoneal mesotheliomas $(n=7)$ or multilocular peritoneal inclusion cysts $(n=6)$ [16]. Similar to adenomatoid tumors of the genital peritoneal mesothelioma. L1CAM staining shows robust staining with the expected membranous pattern in all examined cases of welldifferentiated papillary mesothelioma and adenomatoid tumors of the genital tract, whereas L1CAM expression is absent in normal mesothelial cells and malignant peritoneal mesotheliomas

tract, these data suggest that well-differentiated papillary mesothelioma are also characterized by activated NF-kB signaling, which in well-differentiated papillary mesothelioma can be due to either TRAF7 or CDC42 mutation.

\section{Discussion}

Our study identifies mutually exclusive somatic mutations of TRAF7 and CDC42 in all cases of well-differentiated papillary mesothelioma of the peritoneum. These results confirm that well-differentiated papillary mesothelioma is indeed a clonal neoplasm, and refute the hypothesis that this 
lesion represents reactive mesothelial hyperplasia [14]. Beyond TRAF7 or CDC42 mutation, all tumors had relatively simple genomes with few, if any, additional somatic mutations or chromosomal copy number alterations. The presence of a solitary pathogenic alteration in either TRAF7 or $C D C 42$ in all cases of well-differentiated papillary mesothelioma is in keeping with the low-grade nature of these neoplasms.

We recently identified that adenomatoid tumors of the genital tract are genetically defined by somatic missense mutations in the TRAF7 gene that similarly localize in the WD40 repeats at the $\mathrm{C}$ terminus of the protein [16]. Thus, in addition to sharing a mesothelial cell of origin, both welldifferentiated papillary mesothelioma of the peritoneum and adenomatoid tumors of the genital tract share a common molecular pathogenesis. We thus speculate that welldifferentiated papillary mesothelioma of the peritoneum and adenomatoid tumors of the genital tract may be a single entity that harbors different names depending on the site of origin. In support of this hypothesis are several reported cases of tumors showing mixed features of welldifferentiated papillary mesothelioma and adenomatoid tumor $[6,33]$. Transcriptional or epigenetic profiling of these two tumors is likely to be helpful in clarifying this hypothesis, which may reveal a similar transcriptional and epigenetic landscape if indeed they represent a single entity. Of note though is that a subset of well-differentiated papillary mesothelioma lack TRAF7 mutations and instead harbor $C D C 42$ mutations, whereas all 31 of the adenomatoid tumors of the genital tract harbored TRAF7 mutations and lacked CDC42 mutations in our initial study [16].

The well-differentiated papillary mesothelioma of the peritoneum in this cohort all had intact BAP1 expression and lacked alterations in the BAP1, NF2, CDKN2A, $D D X 3 X, S E T D 2, T P 53$, and $A L K$ genes that are commonly altered in malignant mesotheliomas of the pleural and peritoneal cavities [15, 20-23]. Thus, well-differentiated papillary mesothelioma appears to be genetically distinct from malignant mesothelioma. However, while TRAF7 mutations have not been reported in any cases of malignant peritoneal mesothelioma, they were reportedly seen in a small number of malignant pleural mesotheliomas in one recent study (5 of 216 cases, 2\%) [22]. Two of the five TRAF7-mutant tumors harbored additional BAP1 and TP53 mutations, one harbored additional NF2 mutation, and two lacked additional pathogenic alterations [22]. Whether these cases represent misclassified well-differentiated papillary mesothelioma of the pleural cavity is one possibility, which would be supported by the presence of solitary TRAF7 mutations. Another consideration is that these cases represent well-differentiated papillary mesothelioma that underwent malignant transformation, which would be supported by the presence of TRAF7 mutation combined with other pathogenic alterations such as $B A P 1$ or $N F 2$. Or a third explanation is that TRAF7 mutation can be selected for during tumor development in a small subset of de novo malignant mesotheliomas without progression from a welldifferentiated papillary mesothelioma-like precursor. Further investigation of patients with malignant mesotheliomas harboring TRAF7 mutation is required to understand the biologic nature of these tumors.

In addition to well-differentiated papillary mesothelioma and adenomatoid tumors of the genital tract, TRAF7 mutations are also common in meningiomas and perineuriomas. However, the mechanisms by which TRAF7 mutations contribute to tumor development are not well described. The TRAF7 gene encodes an E3 ubiquitin ligase within the family of TNF-alpha receptor-associated factor (TRAF) proteins and is thought to regulate the NF-kB signaling pathway. We have previously shown that expression of tumor-derived mutant isoforms but not wild-type TRAF7 in cultured human cells causes increased phosphorylation of the p65/RELA subunit of NF-kB, a modification associated with activation, and also upregulation of L1CAM expression, a downstream transcriptional target of NF-kB [16]. Similar to the robust expression of L1CAM seen in adenomatoid tumors harboring TRAF7 mutations, here we show that well-differentiated papillary mesothelioma are also characterized by robust L1CAM expression, indicative of active NF-kB signaling. Robust L1CAM expression was observed in both TRAF7 and CDC42 mutant welldifferentiated papillary mesothelioma, indicating that CDC42 mutations may potentially be an alternative mechanism of driving NF-kB signaling in TRAF7 wild-type mesothelial neoplasms.

CDC42 encodes a small GTPase of the Rho family of proteins belonging to the Ras superfamily of GTPases. CDC42 was first discovered in budding yeast as a temperature sensitive mutant that was defective in budding, while still maintaining the ability to replicate its DNA [34]. CDC42 is now known to be critical for determination of cell polarity and regulation of the actin cytoskeleton including filopodia formation at leading edge during cell motility [35]. Studies have also shown that Rho family GTPases including CDC42 regulate NF-kB signaling and cell cycle progression $[36,37]$. We are currently investigating which of these functions of $C D C 42$ are abrogated by mutations to drive well-differentiated papillary mesothelioma tumorigenesis. Given the presence of heterozygous missense mutations that localize within the GTP binding and effector domains, we anticipate that these are likely to be oncogenic, gain-offunction mutations, as opposed to tumor suppressive, lossof-function variants. While other Rho family GTPases are known to be recurrently mutated in cancer (e.g., $R A C l$ in melanoma and RHOA in gastric adenocarcinoma and angioimmunoblastic $\mathrm{T}$ cell lymphoma [38-42]), CDC42 
has not been identified as a recurrently mutated gene in any human tumor type to date apart from well-differentiated papillary mesothelioma to the best of our knowledge. Why CDC42 mutations are selected for during mesothelioma development, but not any of the other Ras family GTPases is an interesting question that remains to be answered.

In summary, we have characterized the genetic landscape of well-differentiated papillary mesothelioma of the peritoneum that revealed mutually exclusive mutations in TRAF7 and CDC42, along with an absence of genetic alterations that are common in malignant mesothelioma such as BAP1, NF2, CDKN2A, DDX3X, SETD2, TP53, and $A L K$. Furthermore, we show that the combination of BAP1 and L1CAM immunohistochemistry is likely to be a reliable methodology for differentiating well-differentiated papillary mesothelioma from malignant mesothelioma in the majority of cases.

Acknowledgements This study was supported by NIH Director's Early Independence Award (DP5 OD021403) and the UCSF Physician-Scientist Scholar Program to DAS. BCB is supported by an NCI Outstanding Investigator Award (R35 CA220481). We thank the UCSF Brain Tumor Research Center (supported by NIH SPORE Grant P50CA097257) for assistance with L1CAM immunohistochemistry.

\section{Compliance with ethical standards}

Conflict of interest The authors declare that they have no conflict of interest.

\section{References}

1. Foyle A, Al-Jabi M, McCaughey WT. Papillary peritoneal tumors in women. Am J Surg Pathol. 1981;5:241-9.

2. Daya D, McCaughey WT. Well-differentiated papillary mesothelioma of the peritoneum: a clinicopathologic study of 22 cases. Cancer. 1990;65:292-6.

3. Hoekman K, Tognon G, Risse EK, Bloemsma CA, Vermorken JB. Well-differentiated papillary mesothelioma of the peritoneum: a separate entity. Eur J Cancer. 1996;32:255-8.

4. Butnor KJ, Sporn TA, Hammar SP, Roggli VL. Welldifferentiated papillary mesothelioma. Am J Surg Pathol. 2001;25:1304-9.

5. Malpica A, Sant'Ambrogio S, Deavers MT, Silva EG. Welldifferentiated papillary mesothelioma of the female peritoneum: a clinicopathologic study of 26 cases. Am J Surg Pathol. 2012;36:117-27.

6. Chen X, Sheng W, Wang J. Well-differentiated papillary mesothelioma: a clinicopathological and immunohistochemical study of 18 cases with additional observation. Histopathology. 2013;62:805-13.

7. Lee YK, Jun HJ, Nahm JH, et al. Therapeutic strategies for welldifferentiated papillary mesothelioma of the peritoneum. Jpn J Clin Oncol. 2013;43:996-1003.

8. Galateau-Salle F, Vignaud JM, Burke L, et al. Well-differentiated papillary mesothelioma of the pleura: a series of 24 cases. Am J Surg Pathol. 2004;28:534-40.

9. Brimo F, Illei PB, Epstein JI. Mesothelioma of the tunica vaginalis: a series of eight cases with uncertain malignant potential. Mod Pathol. 2010;23:1165-72.
10. Churg A, Allen T, Borczuk AC, et al. Well-differentiated papillary mesothelioma with invasive foci. Am J Surg Pathol. 2014;38:990-8.

11. Baker PM, Clement PB, Young RH. Malignant peritoneal mesothelioma in women: a study of 75 cases with emphasis on their morphologic spectrum and differential diagnosis. Am J Clin Pathol. 2005;123:724-37.

12. Lee M, Alexander HR, Burke A. Diffuse mesothelioma of the peritoneum: a pathological study of 64 tumours treated with cytoreductive therapy. Pathology. 2013;45:464-73.

13. Liu S, Staats P, Lee M, Alexander HR, Burke AP. Diffuse mesothelioma of the peritoneum: correlation between histological and clinical parameters and survival in 73 patients. Pathology. 2014;46:604-9.

14. Baker PM, Clement PB, Young RH. Selected topics in peritoneal pathology. Int J Gynecol Pathol. 2014;33:393-401.

15. Joseph NM, Chen YY, Nasr A, et al. Genomic profiling of malignant peritoneal mesothelioma reveals recurrent alterations in epigenetic regulatory genes BAP1, SETD2, and DDX3X. Mod Pathol. 2017;30:246-54.

16. Goode B, Joseph NM, Stevers M, et al. Adenomatoid tumors of the male and female genital tract are defined by TRAF7 mutations that drive aberrant NF-kB pathway activation. Mod Pathol. 2018;31:660-73.

17. Clark VE, Erson-Omay EZ, Serin A, et al. Genomic analysis of non-NF2 meningiomas reveals mutations in TRAF7, KLF4, AKT1, and SMO. Science. 2013;339:1077-80.

18. Reuss DE, Piro RM, Jones DT, et al. Secretory meningiomas are defined by combined KLF4 K409Q and TRAF7 mutations. Acta Neuropathol. 2013;125:351-8.

19. Klein CJ, Wu Y, Jentoft ME, et al. Genomic analysis reveals frequent TRAF7 mutations in intraneural perineuriomas. Ann Neurol. 2017;81:316-21.

20. Bott M, Brevet M, Taylor BS, et al. The nuclear deubiquitinase BAP1 is commonly inactivated by somatic mutations and 3 p21.1 losses in malignant pleural mesothelioma. Nat Genet. 2011;43:668-72.

21. Alakus H, Yost SE, Woo B, et al. BAP1 mutation is a frequent somatic event in peritoneal malignant mesothelioma. J Transl Med. 2015;13:122.

22. Bueno R, Stawiski EW, Goldstein LD, et al. Comprehensive genomic analysis of malignant pleural mesothelioma identifies recurrent mutations, gene fusions and splicing alterations. Nat Genet. 2016;48:407-16.

23. Hung YP, Dong F, Watkins JC, et al. Identification of ALK rearrangements in malignant peritoneal mesothelioma. JAMA Oncol. 2018;4:235-8.

24. Cigognetti M, Lonardi S, Fisogni S, et al. BAP1 (BRCA1-associated protein 1) is a highly specific marker for differentiating mesothelioma from reactive mesothelial proliferations. Mod Pathol. 2015;28:1043-57.

25. Andrici J, Sheen A, Sioson L, et al. Loss of expression of BAP1 is a useful adjunct, which strongly supports the diagnosis of mesothelioma in effusion cytology. Mod Pathol. 2015;28:1360-8.

26. Leblay N, Lepretre F, Le Stang N, et al. BAP1 is altered by copy number loss, mutation, and/or loss of protein expression in more than $70 \%$ of malignant peritoneal mesotheliomas. J Thorac Oncol. 2017;12:724-33.

27. Cozzi I, Oprescu FA, Rullo E, Ascoli V. Loss of BRCA1associated protein 1 (BAP1) expression is useful in diagnostic cytopathology of malignant mesothelioma in effusions. Diagn Cytopathol. 2018;46:9-14.

28. Pillappa R, Maleszewski JJ, Sukov WR, et al. Loss of BAP1 expression in atypical mesothelial proliferations helps to predict malignant mesothelioma. Am J Surg Pathol. 2018;42:256-63.

29. Lee HE, Molina JR, Sukov WR, Roden AC, Yi ES. BAP1 loss is unusual in well-differentiated papillary mesothelioma and may 
predict development of malignant mesothelioma. Hum Pathol. 2018 May 12 [Epub ahead of print].

30. Zotti T, Scudiero I, Vito P, Stilo R. The emerging role of TRAF7 in tumor development. J Cell Physiol. 2017;232:1233-8.

31. Parker M, Mohankumar KM, Punchihewa C, et al. C11orf95RELA fusions drive oncogenic NF-kB signaling in ependymoma. Nature. 2014;506:451-5.

32. Figarella-Branger D, Lechapt-Zalcman E, Tabouret E, et al. Supratentorial clear cell ependymomas with branching capillaries demonstrate characteristic clinicopathological features and pathological activation of nuclear factor-kappaB signaling. Neuro Oncol. 2016;18:919-27.

33. Hatano Y, Hirose Y, Matsunaga K, et al. Combined adenomatoid tumor and well differentiated papillary mesothelioma of the omentum. Pathol Int. 2011;61:681-5.

34. Johnson DI, Pringle JR. Molecular characterization of CDC42, a Saccharomyces cerevisiae gene involved in the development of cell polarity. J Cell Biol. 1990;111:143-52.

35. Nobes CD, Hall A. Rho, rac, and cdc42 GTPases regulate the assembly of multimolecular focal complexes associated with actin stress fibers, lamellipodia, and filopodia. Cell. 1995;81:53-62.
36. Perona R, Montaner S, Saniger L, Sanchez-Perez I, Bravo R, Lacal JC. Activation of the nuclear factor-kappaB by Rho, CDC42, and Rac-1 proteins. Genes Dev. 1997;11:463-75.

37. Olson MF, Ashworth A, Hall A. An essential role for Rho, Rac, and Cdc42 GTPases in cell cycle progression through G1. Science. 1995;269:1270-2.

38. Hodis E, Watson IR, Kryukov GV, et al. A landscape of driver mutations in melanoma. Cell. 2012;150:251-63.

39. Krauthammer $\mathrm{M}$, Kong $\mathrm{Y}$, $\mathrm{Ha} \mathrm{BH}$, et al. Exome sequencing identifies recurrent somatic RAC1 mutations in melanoma. Nat Genet. 2012;44:1006-14.

40. Sakata-Yanagimoto M, Enami T, Yoshida K, et al. Somatic RHOA mutation in angioimmunoblastic $\mathrm{T}$ cell lymphoma. Nat Genet. 2014;46:171-5.

41. Palomero $\mathrm{T}$, Couronne L, Khiabanian $\mathrm{H}$, et al. Recurrent mutations in epigenetic regulators, RHOA and FYN kinase in peripheral T cell lymphomas. Nat Genet. 2014;46:166-70.

42. Kakiuchi $M$, Nishizawa $T$, Ueda $H$, et al. Recurrent gain-offunction mutations of RHOA in diffuse-type gastric carcinoma. Nat Genet. 2014;46:583-7. 